\title{
Operations of Bipolar Fuzzy Soft Graph
}

\author{
M. Rajeshwari ${ }^{1^{*}}$ \\ Department of Mathematics \\ Presidency University \\ Bangalore, India.
}

\author{
R. Murugesan ${ }^{2}$ \\ Department of Mathematics, \\ Reva University, \\ Bangalore, India.
}

\author{
K. A. Venkatesh ${ }^{3}$ \\ Department of Mathematics and Comp. Science, \\ Myanmar Institute of Information Technology, \\ Myanmar.
}

\begin{abstract}
Numerical demonstrating, investigation and processing of issues with uncertainty is one of the most sizzling regions in interdisciplinary research including connected arithmetic, computational insight and choice sciences. It is significant that uncertainty emerges from different areas has altogether different nature and can't be caught inside a solitary numerical system. In this paper we initiate bipolar fuzzy soft graphs, vertex and edge- induced soft graphs and some operation of bipolar soft fuzzy graph are explore.
\end{abstract}

Keywords-Bipolar fuzzy soft graphs, strong bipolar fuzzy soft graphs, complete bipolar fuzzy soft graphs, regular bipolar fuzzy soft graphs.

\section{INTRODUCTION}

Soft set theory was introduced by Molodtsov[1]. Later Feng, Liu and Fotea combined soft set with fuzzy set and rough set. In 1975[1] Rosenfeld initiated fuzzy graph theory. During the same time various concepts in connectedness with fuzzy graphs was introduced by Yeh and Bang [2]. The concept of bipolar fuzzy graphs was introduced by Akramin. In this paper we initiate bipolar fuzzy soft graphs, vertex and edge- induced soft graphs and some operation of bipolar soft fuzzy graph.

\section{PRELIMINARIES}

Definition 2.1. If $A \subset \mathrm{P}$, then the fuzzy soft set over the universe is a pair of $(M, A)$ where $M: A \rightarrow C^{U}, C^{U}$ is a gathering of fuzzy subset of $U$.

Definition 2.2. [1] Let $S$ be the universal set and $P$ is the set of parameter then $(M, P)$ is called soft set over $\mathrm{S}$ where $\mathrm{M}$ : $\mathrm{P} \rightarrow \mathrm{P}(\mathrm{S})$.

Definition 2.2 [15]: If $A \subset \mathrm{P}$, then the fuzzy soft set over the universe is a pair of $(M, A)$ where $M: A \rightarrow C^{S}, C^{S}$ is a gathering of fuzzy subset of $\mathrm{S}$.

Definition 2.3. If $A \subset \mathrm{P}$, then the bipolar fuzzy soft set over the universe is a pair of $(\mathrm{M}, \mathrm{A})$ where

$M: A \rightarrow B F^{S}$ where $B F^{S}$ is the collection of all bipolar fuzzy subsets of $\mathrm{S}$. It is defined by

$(F, A)=F\left(e_{i}\right)$

$F\left(e_{i}\right)=\left\{c_{i}, \mu^{+}\left(c_{i}\right), \mu^{-}\left(c_{i}\right): \forall\left(c_{i}\right) \in U, \forall\left(e_{i}\right) \in A\right\}$

Definition 2.4. An intersection of two bipolar fuzzy soft sets $(\mathrm{F}, \mathrm{A})$ and $(\mathrm{G}, \mathrm{B})$ is a bipolar fuzzy soft set $(\mathrm{H}, \mathrm{C})$, where $\mathrm{C}=$ $\mathrm{A} \cap \mathrm{B} /=\emptyset$ and $\mathrm{H}: \mathrm{C} \rightarrow \mathrm{BF} \mathrm{U}$ is defined by $\mathrm{H}(\mathrm{e})=\mathrm{F}(\mathrm{e}) \cap$ $\mathrm{G}$ (e) $\forall \mathrm{e} \in \mathrm{C}$ and denoted by $(\mathrm{H}, \mathrm{C})=(\mathrm{F}, \mathrm{A}) \cap^{-}(\mathrm{G}, \mathrm{B})$.

Definition 2.5[4]: Union of two bipolar fuzzy soft sets over a common universe $\mathrm{U}$ is a bipolar fuzzy soft set $(\mathrm{H}, \mathrm{C})$, where $\mathrm{C}=\mathrm{A} \cup \mathrm{B}$ and $H: C \rightarrow B F^{U}$ is defined by

$\mathrm{H}(\mathrm{e})=\mathrm{F}(\mathrm{e})$ if $\mathrm{e} \in \mathrm{A} \backslash \mathrm{B}$

$=\mathrm{G}(\mathrm{e})$ if $\mathrm{e} \in \mathrm{B} \backslash \mathrm{A}$

$=\mathrm{F}(\mathrm{e}) \cup \mathrm{G}(\mathrm{e})$ if $e \in A \cap B$

Denoted as $(F, A) \widetilde{\cup}(G, B)=(H, C)$

\section{MAIN RESULT}

Definition 3.1. An bipolar fuzzy soft graph $\tilde{G}=\left(G^{*}, \tilde{J}_{\varepsilon^{P}, \eta^{N}}, \tilde{L}_{\rho^{P}, \xi^{N}}, A\right)$ is such that

a) $G^{*}=(V, E)$ is a simple graph

b) $A$ is a nonempty set of parameters

c) $\left(\tilde{J}_{\varepsilon^{P}, \eta^{N}}, A\right)$ is a bipolar fuzzy soft set over $V$

d) $\left(\tilde{L}_{\rho^{P}, \xi^{N}}, A\right)$ is a bipolar fuzzy soft set over $E$

e) $\left(\tilde{J}_{\varepsilon^{P}, \eta^{N}}, \tilde{L}_{\rho^{P}, \xi^{N}}\right)$ is a bipolar fuzzy (sub)graph of $G^{*}$ for all $a \in A$. That is

$$
\begin{aligned}
& \tilde{L}_{\rho^{p}}(a)(x y) \leq \min \left\{\tilde{J}_{\varepsilon^{P}}(a)(x), \tilde{J}_{\varepsilon^{P}}(a)(y)\right\} \\
& \tilde{L}_{\zeta^{N}}(a)(x y) \geq \max \left\{\tilde{J}_{\eta^{N}}(a)(x), \tilde{J}_{\eta^{N}}(a)(y)\right\} \forall a \in A ; x, y \in V
\end{aligned}
$$

The bipolar fuzzy soft graph is denoted by $\tilde{B}_{\varsigma, \sigma}(a)$. 
Definition 3.2. An bipolar fuzzy soft graph $\widetilde{G}=\left(G^{*}, \widetilde{J}_{\varepsilon^{P}, \eta^{N}}, \tilde{L}_{\rho^{P}, \xi^{N}}, A\right)$ is said to be vertex induced if $\widetilde{B}_{\zeta, \sigma}(a)=\left(\tilde{J}_{\varepsilon^{P}, \eta^{N}}, \tilde{L}_{\rho^{P}, \xi^{N}}\right)=\tilde{J}_{\varepsilon^{P}, \eta^{N}} \quad \forall a \in A$.

Definition 3.3. An bipolar fuzzy soft graph $\tilde{G}=\left(G^{*}, \tilde{J}_{\varepsilon^{P}, \eta^{N}}, \tilde{L}_{\rho^{P}, \xi^{N}}, A\right)$ is said to be edge induced if $\tilde{B}_{\varsigma, \sigma}(a)=\left(\tilde{J}_{\varepsilon^{P}, \eta^{N}}, \tilde{L}_{\rho^{P}, \xi^{N}}\right)=\tilde{L}_{\rho^{P}, \xi^{N}} \quad \forall a \in A$.

Example3.4.

Consider the bipolar fuzzy graph $G^{*}$. The parameter set is denoted by $A=\left\{e_{1}, e_{3}, e_{5}\right\},\left(\tilde{J}_{\varepsilon^{P}, \eta^{N}}, A\right)$ and $\left(\tilde{L}_{\rho^{P}, \xi^{N}}, A\right)$ is a bipolar fuzzy soft set over $V$ and $E$ respectively, with bipolar fuzzy approximation function $\tilde{J}_{\varepsilon^{P}, \eta^{N}}: A \rightarrow B F^{U}$ and $\tilde{L}_{\rho^{P}, \xi^{N}}: A \rightarrow B F^{U}$

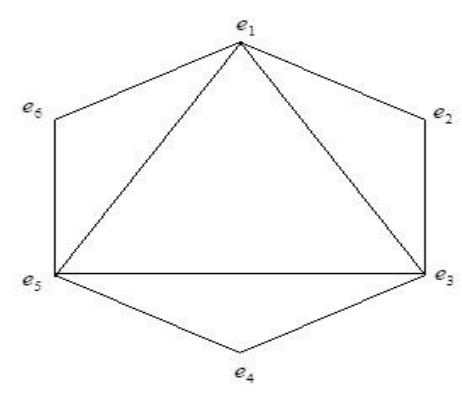

Fig.3.1. Simple Graph $G^{*}$

$\widetilde{J}_{\varepsilon^{p}, \eta^{N}}(a)=\left\{b \in V / a R b \Leftrightarrow \widetilde{d}_{\varepsilon^{p}, \eta^{N}}(a, b)=(1,-1)\right\}$

$\forall a \in A$

i.e. $\tilde{J}_{\varepsilon^{P}, \eta^{N}}\left(e_{1}\right)=\left\{e_{2}, e_{3}, e_{5}, e_{6}\right\}$,

$\tilde{J}_{\varepsilon^{P}, \eta^{N}}\left(e_{3}\right)=\left\{e_{1}, e_{2}, e_{5}, e_{6}\right\}$ and

$\tilde{J}_{\varepsilon^{P}, \eta^{N}}\left(e_{5}\right)=\left\{e_{1}, e_{3}, e_{4}, e_{6}\right\}$

The edges $\tilde{L}_{\rho^{P}, \xi^{N}}(a)=\left\{u v \in E /\{u, v\} \subseteq \tilde{J}_{\varepsilon^{P}, \eta^{N}}(a)\right\}$

$$
\begin{gathered}
\tilde{L}_{\rho^{P}, \xi^{N}}\left(e_{1}\right)=\left\{e_{2} e_{3}, e_{3} e_{5}, e_{5} e_{6}\right\}, \\
\tilde{L}_{\rho^{P}, \xi^{N}}\left(e_{3}\right)=\left\{e_{2} e_{1}, e_{1} e_{5}, e_{5} e_{4}\right\} \text { and } \\
\tilde{L}_{\rho^{P}, \xi^{N}}\left(e_{5}\right)=\left\{e_{4} e_{3}, e_{3} e_{1}, e_{1} e_{6}\right\}
\end{gathered}
$$

Thus,

$$
\begin{aligned}
& \widetilde{B}_{\varsigma^{p}, \sigma^{N}}\left(e_{1}\right)=\left(\widetilde{J}_{\varepsilon^{p}, \eta^{N}}\left(e_{1}\right), \widetilde{L}_{\rho^{p}, \xi^{N}}\left(e_{1}\right)\right), \\
& \widetilde{B}_{\varsigma^{p}, \sigma^{N}}\left(e_{3}\right)=\left(\widetilde{J}_{\varepsilon^{p}, \eta^{N}}\left(e_{3}\right), \widetilde{L}_{\rho^{p}, \xi^{N}}\left(e_{3}\right)\right) \text { and } \\
& \widetilde{B}_{\xi^{p}, \sigma^{N}}\left(e_{5}\right)=\left(\widetilde{J}_{\varepsilon^{p}, \eta^{N}}\left(e_{5}\right), \widetilde{L}_{\rho^{p}, \xi^{N}}\left(e_{5}\right)\right)
\end{aligned}
$$

are bipolar fuzzy soft graphs of $G^{*}$.Tabular representation of vertex and edges of bipolar fuzzy soft graphs.
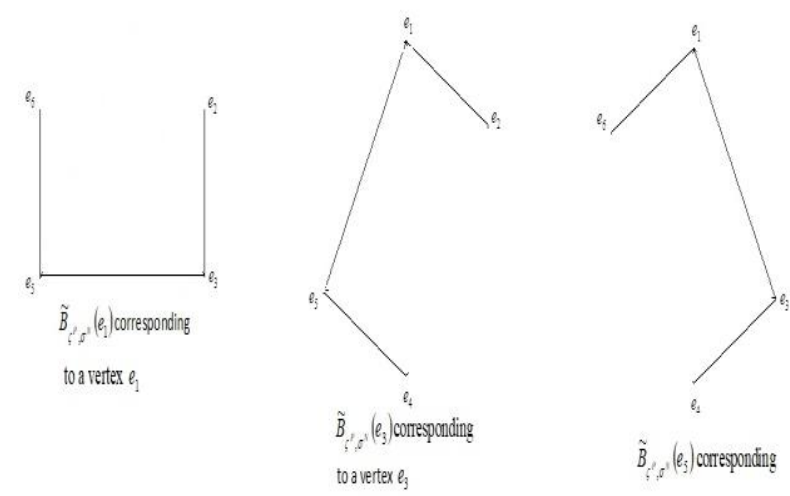

to a vertex $\ell_{\xi}$

Fig.3.2.Subgraphs $\tilde{B}_{\varsigma^{P}, \sigma^{N}}\left(e_{1}\right), \widetilde{B}_{\varsigma^{P}, \sigma^{N}}\left(e_{3}\right), \tilde{B}_{\varsigma^{P}, \sigma^{N}}\left(e_{5}\right)$

\begin{tabular}{|l|l|l|r|r|r|r|}
\hline $\mathrm{A} / \mathrm{V}$ & $e_{1}$ & $e_{2}$ & $e_{3}$ & $e_{4}$ & $e_{5}$ & $e_{6}$ \\
\hline$e_{1}$ & $(0,0)$ & $(1,-1)$ & $(1,-1)$ & $(0,0)$ & $(1,-1)$ & $(1,-1)$ \\
\hline$e_{3}$ & $(1,-1)$ & $(1,-1)$ & $(0,0)$ & $(1,-1)$ & $(1,-1)$ & $(0,0)$ \\
\hline$e_{5}$ & $(1,-1)$ & $(0,0)$ & $(1,-1)$ & $(1,-1)$ & $(0,0)$ & $(1,-1)$ \\
\hline
\end{tabular}

\begin{tabular}{|l|l|l|l|l|l|l|}
\hline $\mathrm{A} / \mathrm{E}$ & $e_{1} e_{2}$ & $e_{2} e_{3}$ & $e_{3} e_{4}$ & $e_{4} e_{5}$ & $e_{5} e_{6}$ & $e_{6} e_{1}$ \\
\hline$e_{1}$ & $(0,0)$ & $(1,-1)$ & $(0,0)$ & $(0,0)$ & $(1,-1)$ & $(0,0)$ \\
\hline$e_{3}$ & $(1,-1)$ & $(0,0)$ & $(0,0)$ & $(1,-1)$ & $(0,0)$ & $(0,0)$ \\
\hline$e_{5}$ & $(0,0)$ & $(0,0)$ & $(1,-1)$ & $(0,0)$ & $(0,0)$ & $(1,-1)$ \\
\hline
\end{tabular}

\begin{tabular}{|l|l|l|l|}
\hline $\mathrm{A} / \mathrm{E}$ & $e_{1} e_{3}$ & $e_{3} e_{5}$ & $e_{5} e_{1}$ \\
\hline$e_{1}$ & $(0,0)$ & $(1,-1)$ & $(0,0)$ \\
\hline$e_{3}$ & $(0,0)$ & $(0,0)$ & $(1,-1)$ \\
\hline$e_{5}$ & $(1,-1)$ & $(0,0)$ & $(0,0)$ \\
\hline
\end{tabular}

Definition3.5.Let $\tilde{G}_{1}=\left\langle\tilde{J}_{\varepsilon_{1}{ }^{P}, \eta_{1}{ }^{N}}, \tilde{L}_{\rho_{1}{ }^{P}, \xi_{1}{ }^{N}}, A\right\rangle$ and $\tilde{G}_{2}=\left\langle\tilde{J}_{\varepsilon_{2}{ }^{P}, \eta_{2}{ }^{N}}, \tilde{L}_{\rho_{2}{ }^{P}, \xi_{2}{ }^{N}}, B\right\rangle$ be the two bipolar fuzzy soft graph .Then $\tilde{G}_{2}$ is the bipolar fuzzy soft subgraph of $\tilde{G}_{1}$. 


\section{$i, B \subseteq A$}

$i i, B_{\beta_{2}^{P}, \delta_{2}^{N}}(x)$ is the subgraphf $B_{\beta_{1}^{P}, \delta_{1}^{N}}(x) \forall x \in B$

Example3.6.Consider the bipolar fuzzy graph $G^{*}$.The parameter of two set is denoted by $A=\left\{e_{1}, e_{2}, e_{5}\right\}$ and $B=\left\{e_{1}, e_{2}\right\},\left(\tilde{J}_{\varepsilon^{P}, \eta^{N}}, A\right)$ and $\left(\tilde{L}_{\rho^{P}, \xi^{N}}, A\right)$ is a bipolar fuzzy soft set over $V$ and E respectively, with bipolar fuzzy approximation function $\quad \widetilde{J}_{\varepsilon^{P}, \eta^{N}}: A \rightarrow B F^{U} \quad$ and $\tilde{L}_{\rho^{P}, \xi^{N}}: A \rightarrow B F^{U}$

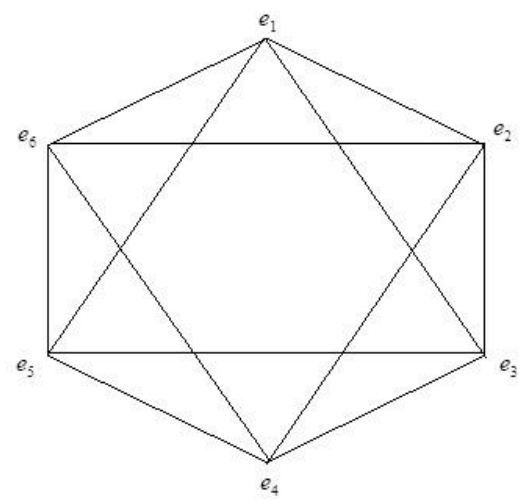

Fig.3.3. Simple Graph $G^{*}$

$\widetilde{J}_{\varepsilon^{p}, \eta^{N}}(a)=\left\{b \in V / a R b \Leftrightarrow \widetilde{d}_{\varepsilon^{p}, \eta^{N}}(a, b) \leq(1,-1)\right\}$

$\forall a \in A$

i.e., $\tilde{J}_{\varepsilon^{p}, \eta^{N}}\left(e_{1}\right)=\left\{e_{1}, e_{2}, e_{3}, e_{5}, e_{6}\right\}$,

$\tilde{J}_{\varepsilon^{P}, \eta^{N}}\left(e_{2}\right)=\left\{e_{1}, e_{2}, e_{3}, e_{4}, e_{6}\right\}$ and

$\tilde{J}_{\varepsilon^{p}, \eta^{N}}\left(e_{5}\right)=\left\{e_{1}, e_{3}, e_{4}, e_{5}, e_{6}\right\}$

The edges

$\tilde{L}_{\rho^{P}, \xi^{N}}(a)=\left\{u v \in E /\{u, v\} \subseteq \tilde{J}_{\varepsilon^{p}, \eta^{N}}(a)\right\} \forall a \in A$

$$
\begin{gathered}
\tilde{L}_{\rho^{P}, \xi^{N}}\left(e_{1}\right)=\left\{e_{1} e_{2}, e_{2} e_{3}, e_{3} e_{5}, e_{5} e_{6}, e_{6} e_{2}, e_{6} e_{1}, e_{5} e_{1}, e_{1} e_{3}\right\}, \\
\tilde{L}_{\rho^{p}, \xi^{N}}\left(e_{2}\right)=\left\{e_{1} e_{2}, e_{2} e_{3}, e_{3} e_{4}, e_{4} e_{6}, e_{6} e_{1}, e_{1} e_{3}, e_{2} e_{4}, e_{2} e_{6}\right\} \text { and } \\
\tilde{L}_{\rho^{p}, \xi^{N}}\left(e_{5}\right)=\left\{e_{1} e_{3}, e_{3} e_{4}, e_{4} e_{5}, e_{5} e_{6}, e_{6} e_{1}, e_{1} e_{5}, e_{5} e_{3}, e_{4} e_{6}\right\}
\end{gathered}
$$
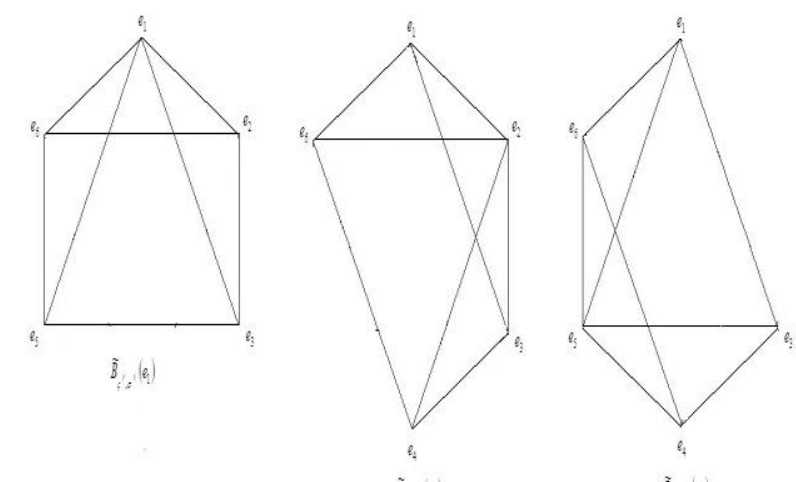

$\tilde{B}_{i, z},\left(\ell_{3}\right)$

Fig.3.4. Subgraphs $\widetilde{B}_{\varsigma^{P}, \sigma^{N}}\left(e_{1}\right), \widetilde{B}_{\varsigma^{P}, \sigma^{N}}\left(e_{2}\right), \widetilde{B}_{\varsigma^{P}, \sigma^{N}}\left(e_{5}\right)$

Now the bipolar fuzzy approximation function $\tilde{J}_{\varepsilon^{P}, \eta^{N}}: B \rightarrow B F^{U}$ and $\tilde{L}_{\rho^{P}, \xi^{N}}: B \rightarrow B F^{U}$

$$
\begin{aligned}
& \widetilde{J}_{\varepsilon^{P}, \eta^{N}}(a)=\left\{b \in V / a R b \Leftrightarrow \widetilde{d}_{\varepsilon^{P}, \eta^{N}}(a, b)=(1,-1)\right\} \\
& \forall a \in B \\
& \text { Then } \widetilde{J}_{\varepsilon^{P}, \eta^{N}}\left(e_{1}\right)=\left\{e_{2}, e_{3}, e_{5}, e_{6}\right\}, \\
& \tilde{J}_{\varepsilon^{P}, \eta^{N}}\left(e_{2}\right)=\left\{e_{1}, e_{3}, e_{4}, e_{6}\right\}
\end{aligned}
$$

$$
\begin{gathered}
\text { The edges } \\
\tilde{L}_{\rho^{P}, \xi^{N}}(a)=\left\{u v \in E /\{u, v\} \subseteq \widetilde{J}_{\varepsilon^{P}, \eta^{N}}(a)\right\} \forall a \in B \\
\tilde{L}_{\rho^{P}, \xi^{N}}\left(e_{1}\right)=\left\{e_{2} e_{3}, e_{3} e_{5}, e_{5} e_{6}, e_{6} e_{2}\right\} \text { and } \\
\tilde{L}_{\rho^{P}, \xi^{N}}\left(e_{2}\right)=\left\{e_{4} e_{6}, e_{6} e_{1}, e_{1} e_{3}, e_{3} e_{4}\right\} . \text { Hence } \\
B \subseteq A \text { and } \\
B_{\beta_{2}^{P}, \delta_{2}^{N}}(a) \text { is the subgraph of } B_{\beta_{1}^{P}, \delta_{1}^{N}}(a) \forall a \in B
\end{gathered}
$$
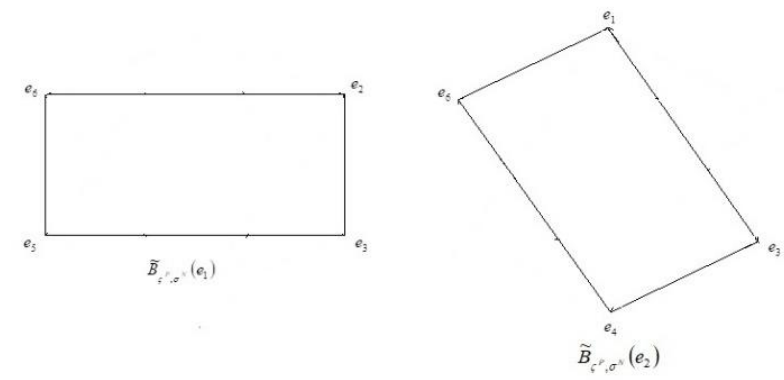

Fig.5 Subgraphs $\widetilde{B}_{\varsigma^{P}, \sigma^{N}}\left(e_{1}\right), \widetilde{B}_{\varsigma^{P}, \sigma^{N}}\left(e_{2}\right)$

Theorem 3.7. Let $\widetilde{G}_{1}=\left\langle\widetilde{J}_{\varepsilon_{1}^{P}, \eta_{1}^{N}}, \widetilde{L}_{\rho_{1}^{P}, \xi_{1}^{N}}, A\right\rangle$ and $\tilde{G}_{2}=\left\langle\tilde{J}_{\varepsilon_{2}{ }^{P}, \eta_{2}{ }^{N}}, \tilde{L}_{\rho_{2}{ }^{P}, \xi_{2}{ }^{N}}, B\right\rangle$ be the two bipolar fuzzy soft graph .Then $\widetilde{G}_{2}$ is the bipolar fuzzy soft subgraph of $\widetilde{G}_{1}$ if 
and only if $\tilde{J}_{\varepsilon_{2}{ }^{P}, \eta_{2}{ }^{N}}(a) \subseteq \tilde{J}_{\varepsilon_{1}{ }^{P}, \eta_{1}{ }^{N}}(a)$ and $\tilde{L}_{\rho_{2}{ }^{P}, \xi_{2}{ }^{N}}(a) \subseteq$ $\tilde{L}_{\rho_{1}^{P}, \xi_{1}^{N}}(a) \forall a \in B$.

Proof:

Suppose $\tilde{G}_{2}$ is the bipolar fuzzy soft subgraph of $\tilde{G}_{1}$.

$$
\begin{aligned}
& i, B \subseteq A \\
& i i, \widetilde{B}_{\varsigma^{P}, \sigma^{N}}\left(e_{2}\right)=\left(\widetilde{J}_{\varepsilon^{P}, \eta^{N}}\left(e_{2}\right), \widetilde{L}_{\rho^{P}, \xi^{N}}\left(e_{2}\right)\right)
\end{aligned}
$$

is the subgraph of

$\widetilde{B}_{\varsigma^{P}, \sigma^{N}}\left(e_{1}\right)=\left(\widetilde{J}_{\varepsilon^{p}, \eta^{N}}\left(e_{1}\right), \widetilde{L}_{\rho^{P}, \xi^{N}}\left(e_{1}\right)\right) \forall a \in B$

i.e., $\tilde{J}_{\varepsilon_{2}{ }^{P}, \eta_{2}{ }^{N}}(a) \subseteq \widetilde{J}_{\varepsilon_{1}{ }^{P}, \eta_{1}{ }^{N}}(a)$ and $\tilde{L}_{\rho_{2}{ }^{P}, \xi_{2}{ }^{N}}(a) \subseteq$

$\tilde{L}_{\rho_{1}^{P}, \xi_{1}^{N}}(a) \forall a \in B$, since

$B_{\beta_{2}^{P}, \delta_{2}^{N}}(a)$ is the subgraph of $B_{\beta_{1}^{P}, \delta_{1}^{N}}(a)$.

Conversely, Assume that $\tilde{J}_{\varepsilon_{2}{ }^{P}, \eta_{2}{ }^{N}}(a) \subseteq \widetilde{J}_{\varepsilon_{1}{ }^{P}, \eta_{1}{ }^{N}}(a)$ and $\tilde{L}_{\rho_{2}{ }^{P}, \xi_{2}{ }^{N}}(a) \subseteq \tilde{L}_{\rho_{1}{ }^{P}, \xi_{1}^{N}}(a) \forall a \in B$. Since $\tilde{G}_{1}$ is the bipolar fuzzy soft graph of $G^{*} . B_{\beta_{1}^{p}, \delta_{1}^{N}}(a)$ is a bipolar fuzzy subgraph of $G^{*}$ for all $a \in A$.

Since $\widetilde{G}_{2}$ is the bipolar fuzzy soft graph of $G^{*} . B_{\beta_{2}^{p}, \delta_{2}^{N}}(a)$ is a bipolar fuzzy subgraph of $G^{*}$ for all $a \in B$. Thus $B_{\beta_{2}^{P}, \delta_{2}^{N}}(a)$ is a bipolar fuzzy subgraph of $B_{\beta_{1}^{P}, \delta_{1}^{N}}(a)$ for all $a \in B$. Hence $\widetilde{G}_{2}$ is the bipolar fuzzy soft subgraph of $\tilde{G}_{1}$.

Definition 3.8. Let $\tilde{G}_{1}=\left\langle\tilde{J}_{\varepsilon_{1}^{P}, \eta_{1}^{N}}, \tilde{L}_{\rho_{1}{ }^{P}, \xi_{1}^{N}}, A\right\rangle \quad$ and $\tilde{G}_{2}=\left\langle\tilde{J}_{\varepsilon_{2}{ }^{P}, \eta_{2}{ }^{N}}, \tilde{L}_{\rho_{2}{ }^{P}, \xi_{2}{ }^{N}}, B\right\rangle$ be the two bipolar fuzzy soft graphs. Extended union of two bipolar fuzzy soft graphs over a common universe $U$ is a bipolar fuzzy soft graph $\left(B_{\beta^{P}, \delta^{N}}, \mathrm{C}\right)$, where $\mathrm{C}=\mathrm{A} \cup \mathrm{B}$ and $B_{\beta^{P}, \delta^{N}}: C \rightarrow B F^{U}$ is defined by

$$
\tilde{J}_{\varepsilon^{P}, \eta^{N}}(e)=\left\{\begin{array}{l}
\tilde{J}_{\varepsilon_{1}{ }^{P}, \eta_{1}{ }^{N}}(e) \text { if } e \in A \backslash B \\
\tilde{J}_{\varepsilon_{2}{ }^{P}, \eta_{2}{ }^{N}}(e) \text { if } e \in B \backslash A \\
\tilde{J}_{\varepsilon_{1}{ }^{P}, \eta_{1}{ }^{N}}(e) \cup \tilde{J}_{\varepsilon_{2}{ }^{P}, \eta_{2}{ }^{N}}(e) \text { if } e \in A \cap B
\end{array}\right.
$$

$$
\tilde{L}_{\rho^{P}, \xi^{N}}(e)=\left\{\begin{array}{l}
\tilde{L}_{\rho_{1}{ }^{P}, \xi_{1}^{N}}(e) \text { if } e \in A \backslash B \\
\tilde{L}_{\rho_{2}{ }^{P}, \xi_{2}{ }^{N}}(e) \text { if } e \in B \backslash A \\
\tilde{L}_{\rho_{1}{ }^{P}, \xi_{1}^{N}}(e) \cup \tilde{L}_{\rho_{2}{ }^{P}, \xi_{2}{ }^{N}}(e) \text { if } e \in A \cap B
\end{array}\right.
$$

i.e.,

$\tilde{G}_{1} \cup_{E} \tilde{G}_{2}=\left\{B_{\beta^{p}, \delta^{N}}(e)=\left(\tilde{J}_{\varepsilon^{p}, \eta^{N}}(e), \tilde{L}_{\rho^{p}, \xi^{N}}(e)\right) / e \in C\right\}$

Example3.9.Consider the bipolar fuzzy graph $G^{*}$.The parameter of two set is denoted by $A=\left\{e_{2}, e_{5}\right\}$ and $B=\left\{e_{1}, e_{4}\right\},\left(\tilde{J}_{\varepsilon^{p}, \eta^{N}}, A\right) \quad$ and $\left(\tilde{L}_{\rho^{P}, \xi^{N}}, A\right)$ is a bipolar fuzzy soft set over $V$ and $\mathrm{E}$ respectively, with bipolar fuzzy approximation function $\tilde{J}_{\varepsilon^{p}, \eta^{N}}: A \rightarrow B F^{U}$ and $\tilde{L}_{\rho^{p}, \xi^{N}}: A \rightarrow B F^{U}$

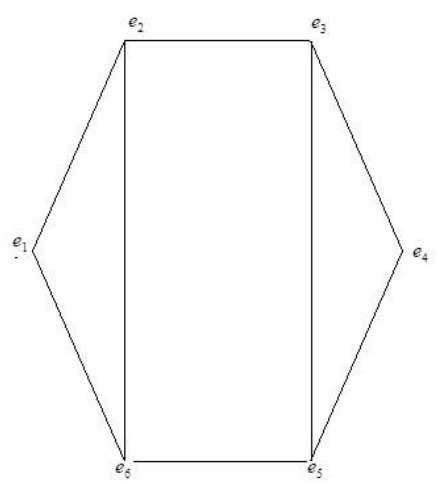

Fig.3.6. Simple Graph $G^{*}$

$\tilde{J}_{\varepsilon^{p}, \eta^{N}}(a)=\left\{b \in V / a R b \Leftrightarrow \tilde{d}_{\varepsilon^{p}, \eta^{N}}(a, b) \leq(1,-1)\right\} \forall a \in A$

i.e., $\tilde{J}_{\varepsilon^{P}, \eta^{N}}\left(e_{2}\right)=\left\{e_{1}, e_{2}, e_{3}, e_{6}\right\}$ and

$\tilde{J}_{\varepsilon^{P}, \eta^{N}}\left(e_{5}\right)=\left\{e_{3}, e_{4}, e_{5}, e_{6}\right\}$

The edges

$$
\begin{gathered}
\tilde{L}_{\rho^{P}, \xi^{N}}(a)=\left\{u v \in E /\{u, v\} \subseteq \tilde{J}_{\varepsilon^{P}, \eta^{N}}(a)\right\} \forall a \in A \\
\tilde{L}_{\rho^{P}, \xi^{N}}\left(e_{2}\right)=\left\{e_{1} e_{2}, e_{2} e_{3}, e_{6} e_{1}, e_{2} e_{6}\right\} \text { and } \\
\tilde{L}_{\rho^{P}, \xi^{N}}\left(e_{5}\right)=\left\{e_{3} e_{4}, e_{4} e_{5}, e_{5} e_{6}, e_{5} e_{3}\right\}
\end{gathered}
$$




$$
\begin{aligned}
\widetilde{B}_{\xi^{p}, \sigma^{N}}\left(e_{2}\right) & =\left(\widetilde{J}_{\varepsilon^{p}, \eta^{N}}\left(e_{3}\right), \widetilde{L}_{\rho^{p}, \xi^{N}}\left(e_{3}\right)\right) \text { and } \\
\widetilde{B}_{\xi^{p}, \sigma^{N}} & \left(e_{5}\right)=\left(\widetilde{J}_{\varepsilon^{p}, \eta^{N}}\left(e_{5}\right), \widetilde{L}_{\rho^{p}, \xi^{N}}\left(e_{5}\right)\right)
\end{aligned}
$$

Now the bipolar fuzzy approximation function $\tilde{J}_{\varepsilon^{P}, \eta^{N}}: B \rightarrow B F^{U} \quad$ and $\quad \tilde{L}_{\rho^{P}, \xi^{N}}: B \rightarrow B F^{U}$ $\tilde{J}_{\varepsilon^{P}, \eta^{N}}(a)=\left\{b \in V / a R b \Leftrightarrow \tilde{d}_{\varepsilon^{P}, \eta^{N}}(a, b)=(1,-1)\right\} \forall a \in B$

$$
\text { Then } \tilde{J}_{\varepsilon^{P}, \eta^{N}}\left(e_{1}\right)=\left\{e_{2}, e_{6}\right\} \text { and } \tilde{J}_{\varepsilon^{P}, \eta^{N}}\left(e_{4}\right)=\left\{e_{3}, e_{5}\right\}
$$

The edges

$$
\begin{gathered}
\widetilde{L}_{\rho^{P}, \xi^{N}}(a)=\left\{u v \in E /\{u, v\} \subseteq \widetilde{J}_{\varepsilon^{P}, \eta^{N}}(a)\right\} \forall a \in B \\
\widetilde{L}_{\rho^{P}, \xi^{N}}\left(e_{1}\right)=\left\{e_{6} e_{2}\right\} \text { and } \widetilde{L}_{\rho^{P}, \xi^{N}}\left(e_{4}\right)=\left\{e_{3} e_{5}\right\} \\
\widetilde{B}_{\varsigma^{P}, \sigma^{N}}\left(e_{1}\right)=\left(\widetilde{J}_{\varepsilon^{P}, \eta^{N}}\left(e_{1}\right), \widetilde{L}_{\rho^{P}, \xi^{N}}\left(e_{1}\right)\right) \text { and } \\
\widetilde{B}_{\varsigma^{P}, \sigma^{N}}\left(e_{4}\right)=\left(\widetilde{J}_{\varepsilon^{P}, \eta^{N}}\left(e_{4}\right), \widetilde{L}_{\rho^{P}, \xi^{N}}\left(e_{4}\right)\right)
\end{gathered}
$$

Extended union of two bipolar fuzzy soft graphs

$$
\widetilde{G}_{1} \cup_{E} \widetilde{G}_{2}=\left\{B_{\beta^{P}, \delta^{N}}(e)=\left(\tilde{J}_{\varepsilon^{P}, \eta^{N}}(e), \tilde{L}_{\rho^{P}, \xi^{N}}(e)\right) / e \in C\right\}
$$$$
\text { Where } \mathrm{C}=\mathrm{A} \cup \mathrm{B}=\left\{e_{1}, e_{2}, e_{4}, e_{5}\right\}
$$

$$
\begin{aligned}
& \tilde{J}_{\varepsilon^{P}, \eta^{N}}\left(e_{1}\right)=\left\{e_{2}, e_{6}\right\}, \tilde{L}_{\rho^{P}, \xi^{N}}\left(e_{1}\right)=\left\{e_{6} e_{2}\right\} \\
& \tilde{J}_{\varepsilon^{P}, \eta^{N}}\left(e_{2}\right)=\left\{e_{1}, e_{2}, e_{3}, e_{6}\right\}, \\
& \tilde{L}_{\rho^{P}, \xi^{N}}\left(e_{2}\right)=\left\{e_{1} e_{2}, e_{2} e_{3}, e_{6} e_{1}, e_{2} e_{6}\right\} \\
& \tilde{J}_{\varepsilon^{P}, \eta^{N}}\left(e_{4}\right)=\left\{e_{3}, e_{5}\right\}, \tilde{L}_{\rho^{P}, \xi^{N}}\left(e_{4}\right)=\left\{e_{3} e_{5}\right\} \\
& \tilde{J}_{\varepsilon^{P}, \eta^{N}}\left(e_{5}\right)=\left\{e_{3}, e_{4}, e_{5}, e_{6}\right\} \\
& \tilde{L}_{\rho^{P}, \xi^{N}}\left(e_{5}\right)=\left\{e_{3} e_{4}, e_{4} e_{5}, e_{5} e_{6}, e_{5} e_{3}\right\}
\end{aligned}
$$
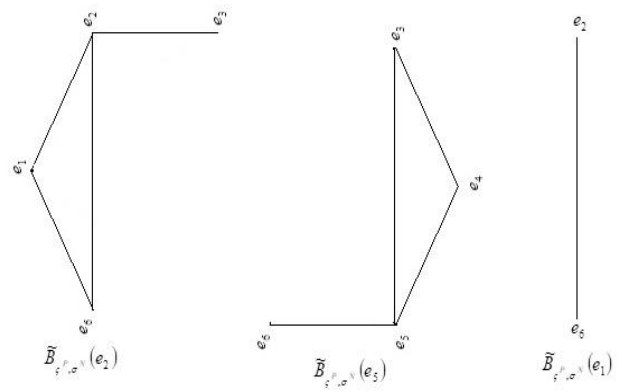

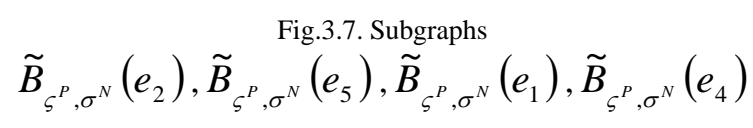

Then subgraphs of $G^{*}$

$$
\begin{aligned}
& \widetilde{B}_{\xi^{p}, \sigma^{N}}\left(e_{1}\right)=\left(\widetilde{J}_{\varepsilon^{p}, \eta^{N}}\left(e_{1}\right), \widetilde{L}_{\rho^{p}, \xi^{N}}\left(e_{1}\right)\right), \\
& \widetilde{B}_{S^{p}, \sigma^{N}}\left(e_{2}\right)=\left(\widetilde{J}_{\varepsilon^{p}, \eta^{N}}\left(e_{2}\right), \widetilde{L}_{\rho^{p}, \xi^{N}}\left(e_{2}\right)\right), \\
& \widetilde{B}_{\xi^{p}, \sigma^{N}}\left(e_{4}\right)=\left(\widetilde{J}_{\varepsilon^{p}, \eta^{N}}\left(e_{4}\right), \widetilde{L}_{\rho^{p}, \xi^{N}}\left(e_{4}\right)\right) \text { and } \\
& \widetilde{B}_{\xi^{p}, \sigma^{N}}\left(e_{5}\right)=\left(\widetilde{J}_{\varepsilon^{p}, \eta^{N}}\left(e_{5}\right), \widetilde{L}_{\rho^{p}, \xi^{N}}\left(e_{5}\right)\right) \\
& \text { Therefore } \\
& \widetilde{G}_{1} \cup_{E} \widetilde{G}_{2}=\left\{B_{\beta^{p}, \delta^{N}}\left(e_{1}\right), B_{\beta^{p}, \delta^{N}}\left(e_{2}\right), B_{\beta^{p}, \delta^{N}}\left(e_{4}\right), B_{\beta^{p}, \delta^{N}}\left(e_{5}\right)\right\}
\end{aligned}
$$

Theorem 3.10. Let $\tilde{G}_{1}=\left\langle\tilde{J}_{\varepsilon_{1}{ }^{P}, \eta_{1}{ }^{N}}, \tilde{L}_{\rho_{1}{ }^{P}, \xi_{1}}, A\right\rangle$ and $\tilde{G}_{2}=\left\langle\tilde{J}_{\varepsilon_{2}{ }^{P}, \eta_{2}{ }^{N}}, \tilde{L}_{\rho_{2}{ }^{P}, \xi_{2}{ }^{N}}, B\right\rangle$ be the two bipolar fuzzy soft graphs with $A \cap B \neq \phi$ and $\tilde{J}_{\varepsilon_{1}{ }^{P}, \eta_{1}{ }^{N}}(a) \cap \tilde{J}_{\varepsilon_{2}{ }^{P}, \eta_{2}{ }^{N}}(a) \neq \phi$ for all $a \in A \cap B$. Then their union is the bipolar fuzzy soft graph of $G^{*}$. Proof:

Let $\tilde{G}_{1}=\left\langle\tilde{J}_{\varepsilon_{1}{ }^{P}, \eta_{1}{ }^{N}}, \tilde{L}_{\rho_{1}{ }^{P}, \xi_{1}{ }^{N}}, A\right\rangle$ and $\tilde{G}_{2}=\left\langle\tilde{J}_{\varepsilon_{2}{ }^{P}, \eta_{2}{ }^{N}}, \tilde{L}_{\rho_{2}{ }^{P}, \xi_{2}{ }^{N}}, B\right\rangle$ be the two bipolar fuzzy soft graphs. Extended union of two bipolar fuzzy soft graphs $\widetilde{G}_{1} \cup_{E} \widetilde{G}_{2}=\left\{B_{\beta^{P}, \delta^{N}}(e)=\left(\widetilde{J}_{\varepsilon^{P}, \eta^{N}}(e), \tilde{\tilde{L}}_{\rho^{P}, \xi^{N}}(e)\right) / e \in C\right\}$

Where $\mathrm{C}=\mathrm{A} \cup \mathrm{B}$

$$
\begin{aligned}
\tilde{J}_{\varepsilon^{P}, \eta^{N}}(e)= & \left\{\begin{array}{l}
\tilde{J}_{\varepsilon_{1}{ }^{P}, \eta_{1}{ }^{N}}(e) \text { if } e \in A \backslash B \\
\tilde{J}_{\varepsilon_{2}{ }^{P}, \eta_{2}{ }^{N}}(e) \text { if } e \in B \backslash A \\
\tilde{J}_{\varepsilon_{1}{ }^{P}, \eta_{1}{ }^{N}}(e) \cup \tilde{J}_{\varepsilon_{2}{ }^{P}, \eta_{2}{ }^{N}}(e) \text { if } e \in A \cap B
\end{array}\right. \\
\tilde{L}_{\rho^{P}, \xi^{N}}(e)= & \left\{\begin{array}{l}
\tilde{L}_{\rho_{1}{ }^{P}, \xi_{1}}{ }^{N} \\
\tilde{L}_{\rho_{2}{ }^{P}, \xi_{2}{ }^{N}}(e) \text { if } e \in A \backslash B \\
\tilde{L}_{\rho_{1}{ }^{P}, \xi_{1}{ }^{N}}(e) \cup \tilde{L}_{\rho_{2}{ }^{P}, \xi_{2}{ }^{N}}(e) \text { if } e \in B \backslash A \cap B
\end{array}\right.
\end{aligned}
$$

Since $\widetilde{G}_{1}$ is the bipolar fuzzy soft graph of $G^{*} \cdot B_{\beta_{1}^{P}, \delta_{1}^{N}}(a)$ is a connected bipolar fuzzy subgraph of $G^{*}$ for all $a \in A \backslash B$. Since $\tilde{G}_{2}$ is the bipolar fuzzy soft graph of $G^{*} . B_{\beta_{2}^{P}, \delta_{2}^{N}}(a)$ is a connected bipolar fuzzy subgraph of $G^{*}$ for all $a \in B \backslash A$. Let $a \in A \cap B$ and

$$
\left(\widetilde{J}_{\varepsilon^{P}, \eta^{N}}(a), \widetilde{L}_{\rho^{P}, \xi^{N}}(a)\right)=\left(\begin{array}{l}
\widetilde{J}_{\varepsilon_{1}{ }^{P}, \eta_{1}{ }^{N}}(a) \cup \widetilde{J}_{\varepsilon_{2}{ }^{P}, \eta_{2}{ }^{N}}(a), \\
\widetilde{L}_{\rho_{1}{ }^{P}, \xi_{1}{ }^{N}}(a) \cup \widetilde{L}_{\rho_{2}{ }^{P}, \xi_{2}{ }^{N}}(a)
\end{array}\right) \text {. }
$$


Since $\left(\tilde{J}_{\varepsilon_{1}{ }^{P}, \eta_{1}^{N}}(a), \tilde{L}_{\rho_{1}^{P}, \xi_{1}^{N}}(a)\right)$ and

$\left(\tilde{J}_{\varepsilon_{2}{ }^{P}, \eta_{2}{ }^{N}}(a), \tilde{L}_{\rho_{2}{ }^{P}, \xi_{2}{ }^{N}}(a)\right)$ are connected bipolar fuzzy

subgraph of $G^{*}$ by assumption

$\widetilde{J}_{\varepsilon_{1}{ }^{P}, \eta_{1}{ }^{N}}(a) \cap \widetilde{J}_{\varepsilon_{2}{ }^{P}, \eta_{2}{ }^{N}}(a) \neq \phi$ for all $a \in A \cap B$.

$\left(\tilde{J}_{\varepsilon^{P}, \eta^{N}}(a), \tilde{L}_{\rho^{P}, \xi^{N}}(a)\right)$ is a connected bipolar fuzzy

subgraph of $G^{*}$ and

$\widetilde{G}_{1} \cup_{E} \widetilde{G}_{2}=\left\{B_{\beta^{P}, \delta^{N}}(e)=\left(\widetilde{J}_{\varepsilon^{P}, \eta^{N}}(e), \tilde{L}_{\rho^{P}, \xi^{N}}(e)\right) / e \in C\right\}$ is a bipolar soft fuzzy graph of $G^{*}$.

Definition 3.11. Let $\tilde{G}_{1}=\left\langle\tilde{J}_{\varepsilon_{1}^{P}, \eta_{1}^{N}}, \tilde{L}_{\rho_{1}^{P}, \xi_{1}^{N}}, A\right\rangle$ and $\tilde{G}_{2}=\left\langle\widetilde{J}_{\varepsilon_{2}{ }^{P}, \eta_{2}{ }^{N}}, \tilde{L}_{\rho_{2}{ }^{P}, \xi_{2}{ }^{N}}, B\right\rangle$ be the two bipolar fuzzy soft graphs. Restricted union of two bipolar fuzzy soft graphs over a common universe $\mathrm{U}$ is a bipolar fuzzy soft graph $\left(B_{\beta^{P}, \delta^{N}}, \mathrm{C}\right)$, where $\mathrm{C}=A \cap B$ and $B_{\beta^{P}, \delta^{N}}: C \rightarrow B F^{U}$ is defined by

$\left(\widetilde{J}_{\varepsilon^{P}, \eta^{N}}(a), \widetilde{L}_{\rho^{P}, \xi^{N}}(a)\right)=\left(\begin{array}{l}\widetilde{J}_{\varepsilon_{1}^{P}, \eta_{1}^{N}}(a) \cup \widetilde{J}_{\varepsilon_{2}{ }^{P}, \eta_{2}^{N}}(a), \\ \widetilde{L}_{\rho_{1}{ }^{P}, \xi_{1}^{N}}(a) \cup \widetilde{L}_{\rho_{2}{ }^{P}, \xi_{2}{ }^{N}}(a)\end{array}\right)$

Definition 3.12. Let $\tilde{G}_{1}=\left\langle\tilde{J}_{\varepsilon_{1}{ }^{P}, \eta_{1}^{N}}, \tilde{L}_{\rho_{1}^{P}, \xi_{1}^{N}}, A\right\rangle$ and $\tilde{G}_{2}=\left\langle\widetilde{J}_{\varepsilon_{2}{ }^{P}, \eta_{2}{ }^{N}}, \tilde{L}_{\rho_{2}{ }^{P}, \xi_{2}{ }^{N}}, B\right\rangle$ be the two bipolar fuzzy soft graphs. Extended intersection of two bipolar fuzzy soft graphs over a common universe $\mathrm{U}$ is a bipolar fuzzy soft graph $\left(B_{\beta^{P}, \delta^{N}}, \mathrm{C}\right), \quad$ where $\mathrm{C}=A \cup B$ and $B_{\beta^{P}, \delta^{N}}: C \rightarrow B F^{U}$ is defined by

$\tilde{J}_{\varepsilon^{P}, \eta^{N}}(e)=\left\{\begin{array}{l}\tilde{J}_{\varepsilon_{1}^{p}, \eta_{1}^{N}}(e) \text { if } e \in A \backslash B \\ \tilde{J}_{\varepsilon_{2}^{P}, \eta_{2}^{N}}(e) \text { if } e \in B \backslash A \\ \tilde{J}_{\varepsilon_{1}{ }^{p}, \eta_{1}^{N}}(e) \cap \tilde{J}_{\varepsilon_{2}^{p}, \eta_{2}{ }^{N}}(e) \text { if } e \in A \cap B\end{array}\right.$

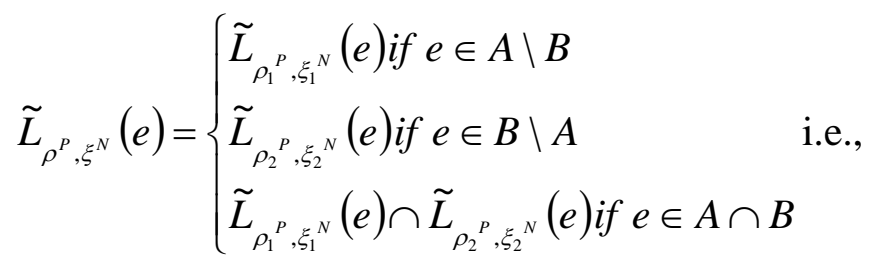

$\tilde{G}_{1} \cap_{E} \tilde{G}_{2}=\left\{B_{\beta^{P}, \delta^{N}}(e)=\left(\tilde{J}_{\varepsilon^{P}, \eta^{N}}(e), \tilde{L}_{\rho^{P}, \xi^{N}}(e)\right) / e \in C\right\}$
Example3.13.Consider the bipolar fuzzy graph $G^{*}$.The parameter of two set is denoted by $A=\left\{e_{1}, e_{2}, e_{5}\right\}$ and $B=\left\{e_{1}, e_{2}\right\},\left(\tilde{J}_{\varepsilon^{P}, \eta^{N}}, A\right)$ and $\left(\tilde{L}_{\rho^{P}, \xi^{N}}, A\right)$ is a bipolar fuzzy soft set over $V$ and $\mathrm{E}$ respectively, with bipolar fuzzy approximation function $\quad \widetilde{J}_{\varepsilon^{P}, \eta^{N}}: A \rightarrow B F^{U} \quad$ and $\tilde{L}_{\rho^{P}, \xi^{N}}: A \rightarrow B F^{U}$

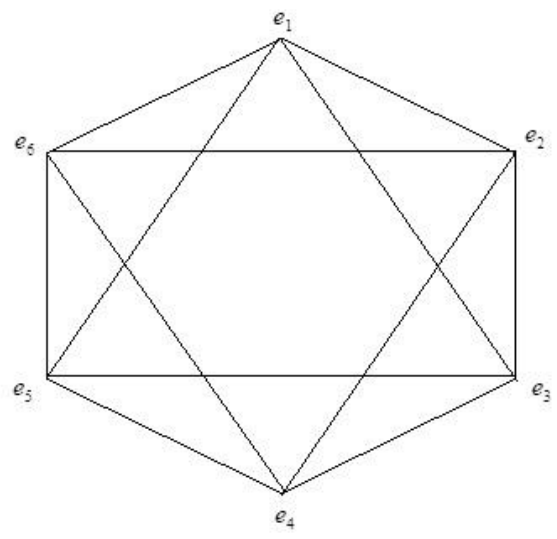

Fig.3.8. Simple Graph $G^{*}$

$\tilde{J}_{\varepsilon^{p}, \eta^{N}}(a)=\left\{b \in V / a R b \Leftrightarrow \tilde{d}_{\varepsilon^{p}, \eta^{N}}(a, b)=\operatorname{rad}\left(G^{*}\right)\right\} \forall a \in A$ i.e.,

$\widetilde{J}_{\varepsilon^{P}, \eta^{N}}\left(e_{1}\right)=\left\{e_{2}, e_{3}, e_{5}, e_{6}\right\}$,

$\tilde{J}_{\varepsilon^{p}, \eta^{N}}\left(e_{2}\right)=\left\{e_{1}, e_{3}, e_{4}, e_{6}\right\}$ and

$\tilde{J}_{\varepsilon^{P}, \eta^{N}}\left(e_{5}\right)=\left\{e_{1}, e_{3}, e_{4}, e_{6}\right\}$

The edges

$\tilde{L}_{\rho^{P}, \xi^{N}}(a)=\left\{u v \in E /\{u, v\} \subseteq \widetilde{J}_{\varepsilon^{p}, \eta^{N}}(a)\right\} \forall a \in A$

$\tilde{L}_{\rho^{P}, \xi^{N}}\left(e_{1}\right)=\left\{e_{2} e_{3}, e_{3} e_{5}, e_{5} e_{6}, e_{6} e_{2}\right\}$,

$\tilde{L}_{\rho^{P}, \xi^{N}}\left(e_{2}\right)=\left\{e_{3} e_{4}, e_{4} e_{6}, e_{6} e_{1}, e_{1} e_{3}\right\}$ and

$\tilde{L}_{\rho^{P}, \xi^{N}}\left(e_{5}\right)=\left\{e_{1} e_{3}, e_{3} e_{4}, e_{6} e_{1}, e_{4} e_{6}\right\}$ 

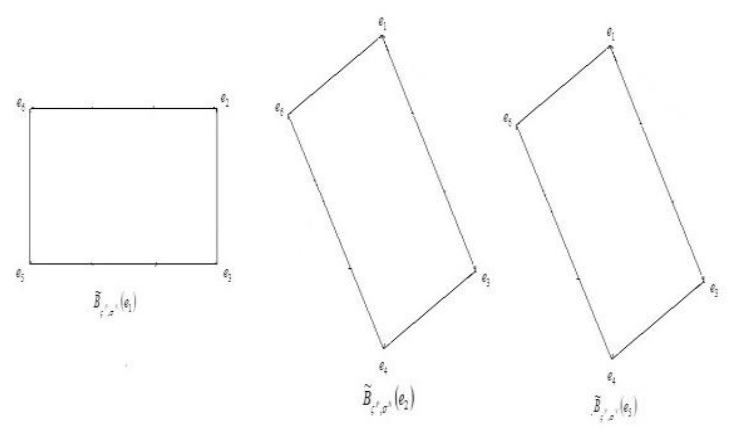

Fig.3.9. Subgraphs $\widetilde{B}_{\varsigma^{P}, \sigma^{N}}\left(e_{1}\right), \widetilde{B}_{\varsigma^{P}, \sigma^{N}}\left(e_{2}\right), \widetilde{B}_{\varsigma^{P}, \sigma^{N}}\left(e_{5}\right)$

Now the bipolar fuzzy approximation function $\widetilde{J}_{\varepsilon^{p}, \eta^{N}}: B \rightarrow B F^{U}$ and $\tilde{L}_{\rho^{p}, \xi^{N}}: B \rightarrow B F^{U}$

$\widetilde{J}_{\varepsilon^{p}, \eta^{N}}(a)=\left\{b \in V / a R b \Leftrightarrow \widetilde{d}_{\varepsilon^{p}, \eta^{N}}(a, b) \leq \operatorname{rad}\left(G^{*}\right)\right\}$ $\forall a \in B$

Then

$$
\begin{aligned}
& \tilde{J}_{\varepsilon^{p}, \eta^{N}}\left(e_{1}\right)=\left\{e_{1}, e_{2}, e_{3}, e_{5}, e_{6}\right\}, \\
& \widetilde{J}_{\varepsilon^{p}, \eta^{N}}\left(e_{2}\right)=\left\{e_{1}, e_{2}, e_{3}, e_{4}, e_{6}\right\}
\end{aligned}
$$

The edges

$\tilde{L}_{\rho^{p}, \xi^{N}}(a)=\left\{u v \in E /\{u, v\} \subseteq \widetilde{J}_{\varepsilon^{p}, \eta^{N}}(a)\right\} \forall a \in B$

$\tilde{L}_{\rho^{p}, \xi^{\xi^{*}}}\left(e_{1}\right)=\left\{e_{1} e_{2}, e_{2} e_{3}, e_{3} e_{5}, e_{5} e_{6}, e_{6} e_{2}, e_{6} e_{1}, e_{5} e_{1}, e_{1} e_{3}\right\}$ and

$\tilde{L}_{\rho^{p}, \xi^{*}}\left(e_{2}\right)=\left\{e_{1} e_{2}, e_{2} e_{3}, e_{4} e_{3}, e_{4} e_{6}, e_{6} e_{1}, e_{2} e_{4}, e_{2} e_{6}, e_{1} e_{3}\right\}$

The extended intersection of two bipolar soft fuzzy graph is $\widetilde{G}_{1} \cap_{E} \widetilde{G}_{2}=\left\{B_{\beta^{p}, \delta^{N}}(e)=\left(\widetilde{J}_{\varepsilon^{p}, \eta^{N}}(e), \widetilde{L}_{\rho^{p}, \xi^{N}}(e)\right) / e \in C\right\}$ where $\mathrm{C}=A \cup B$ $\widetilde{J}_{\varepsilon^{p}, \eta^{N}}\left(e_{1}\right)=\left\{e_{1}, e_{2}, e_{3}, e_{5}, e_{6}\right\}$, $\tilde{L}_{\rho^{p}, \xi^{N}}\left(e_{1}\right)=\left\{e_{1} e_{2}, e_{2} e_{3}, e_{3} e_{5}, e_{5} e_{6}, e_{6} e_{2}, e_{6} e_{1}, e_{5} e_{1}, e_{1} e_{3}\right\}$ $\widetilde{J}_{\varepsilon^{p}, \eta^{N}}\left(e_{2}\right)=\left\{e_{1}, e_{2}, e_{3}, e_{4}, e_{6}\right\}$, $\tilde{L}_{\rho^{p}, \xi^{k^{*}}}\left(e_{2}\right)=\left\{e_{1} e_{2}, e_{2} e_{3}, e_{4} e_{3}, e_{4} e_{6}, e_{6} e_{1}, e_{2} e_{4}, e_{2} e_{6}, e_{1} e_{3}\right\}$ and

$$
\begin{gathered}
\tilde{J}_{\varepsilon^{p}, \eta^{N}}\left(e_{5}\right)=\left\{e_{1}, e_{3}, e_{4}, e_{6}\right\}, \\
\tilde{L}_{\rho^{p}, \xi^{N}}\left(e_{5}\right)=\left\{e_{1} e_{3}, e_{3} e_{4}, e_{6} e_{1}, e_{4} e_{6}\right\} \text { Finally, complete }
\end{gathered}
$$
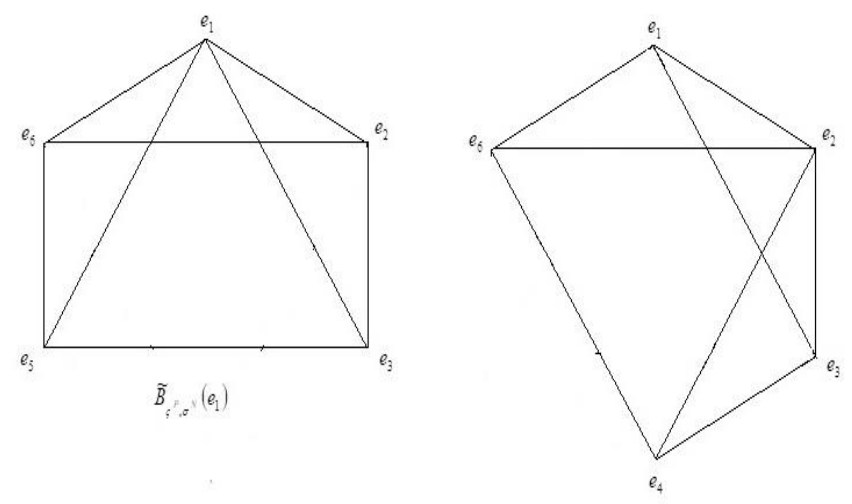

$\tilde{B}_{i, g}\left(e_{2}\right)$

Fig.3.10. Subgraphs $\tilde{B}_{\varsigma^{P}, \sigma^{N}}\left(e_{1}\right), \widetilde{B}_{\varsigma^{P}, \sigma^{N}}\left(e_{2}\right)$

Then subgraphs of $G^{*}$

$$
\begin{aligned}
& \widetilde{B}_{\varsigma^{p}, \sigma^{N}}\left(e_{1}\right)=\left(\widetilde{J}_{\varepsilon^{p}, \eta^{N}}\left(e_{1}\right), \widetilde{L}_{\rho^{p}, \xi^{N}}\left(e_{1}\right)\right), \\
& \widetilde{B}_{\varsigma^{p}, \sigma^{N}}\left(e_{2}\right)=\left(\widetilde{J}_{\varepsilon^{p}, \eta^{N}}\left(e_{2}\right), \widetilde{L}_{\rho^{p}, \xi^{N}}\left(e_{2}\right)\right) \text { and } \\
& \widetilde{B}_{\varsigma^{p}, \sigma^{N}}\left(e_{5}\right)=\left(\widetilde{J}_{\varepsilon^{p}, \eta^{N}}\left(e_{5}\right), \widetilde{L}_{\rho^{p}, \xi^{N}}\left(e_{5}\right)\right)
\end{aligned}
$$

Therefore

$$
\tilde{G}_{1} \cap E \tilde{G}_{2}=\left\{B_{\beta^{P}, \delta^{N}}\left(e_{1}\right), B_{\beta^{P}, \delta^{N}}\left(e_{2}\right), B_{\beta^{P}, \delta^{N}}\left(e_{5}\right)\right\}
$$

Definition 3.14. Let $\tilde{G}_{1}=\left\langle\tilde{J}_{\varepsilon_{1}^{P}, \eta_{1}^{N}}, \tilde{L}_{\rho_{1}^{P}, \xi_{1}^{N}}, A\right\rangle$ and $\tilde{G}_{2}=\left\langle\tilde{J}_{\varepsilon_{2}{ }^{P}, \eta_{2}{ }^{N}}, \tilde{L}_{\rho_{2}{ }^{P}, \xi_{2}{ }^{N}}, B\right\rangle$ be the two bipolar fuzzy soft graphs. Restricted intersection of two bipolar fuzzy soft graphs over a common universe $\mathrm{U}$ is such that $A \cap B=\phi$ bipolar fuzzy soft graph $\left(B_{\beta^{P}, \delta^{N}}, \mathrm{C}\right)$, where $\mathrm{C}=A \cap B$ and $B_{\beta^{P}, \delta^{N}}: C \rightarrow B F^{U}$ is defined by

$$
\left(\widetilde{J}_{\varepsilon^{P}, \eta^{N}}(a), \widetilde{L}_{\rho^{P}, \xi^{N}}(a)\right)=\left(\begin{array}{l}
\widetilde{J}_{\varepsilon_{1}{ }^{P}, \eta_{1}{ }^{N}}(a) \cap \widetilde{J}_{\varepsilon_{2}{ }^{P}, \eta_{2}{ }^{N}}(a), \\
\widetilde{L}_{\rho_{1}{ }^{P}, \xi_{1}{ }^{N}}(a) \cap \widetilde{L}_{\rho_{2}{ }^{P}, \xi_{2}{ }^{N}}(a)
\end{array}\right)
$$

\section{REFERENCES}

[1] A. Rosenfeld, "Fuzzy graphs", in: L.A. Zadeh, K.S. Fu, K Tanaka and M. Shimura,(editors), Fuzzy sets and its application to cognitive and decision process, Academic press, New York (1975) pp. 77 - 95.

[2] R.T.Yeh and S.Y.Bang, "Fuzzy relations, fuzzy graphs and their applications to clustering analysis", in: L.A. Zadeh, K.S. Fu, K Tanaka and M. Shimura,(editors), Fuzzy sets and its application to cognitive and decision process, Academic press, New York (1975) $125-149$.

[3] A. Aygünoglu, H. Aygün, "Introduction to fuzzy soft groups", Computers and Mathematics with Applications 58(2009), 1279 1286.

[4] P.K.Maji, A.R.Roy, R.Biswas, "On intuitionistic fuzzy soft sets", The journal of fuzzy mathematics 12(2004), 669-683. 
[5] Saleem Abdullah, Muhammad Aslam and Kifayat Ullah, "Bipolar Fuzzy Soft sets and its applications in decision making problem", Journal of Intelligent and Fuzzy, March 2014

[6] T. Pathinathan and J. Jesintha Rosline 2014 "Matrix Representation of Double layered fuzzy graph and its properties", Annals of Pure and Applied Mathematics, Vol. 8, No. 2, pp. 51 58

[7] Muhammad Akram, Saira Nawaz, "on fuzzy soft graphs", Italian journal of pure and applied mathematics, 34(2015), 497-514.

[8] Xu, W., Ma, J., Wang, S., Hao, G., "Vague soft sets and their properties", Computers and Mathematics with Applications 59(2010), 787-794.
[9] M.Rajeshwari, R. Murugesan, K. A. Venkatesh, "Properties of Bipolar fuzzy soft graphs", International Journal of Mechanical Engineering and Technology 9(13) 2018,pp.537-546.

[10] A.Nagoorgani and K.Radha, "The degree of a vertex in some fuzzy graphs", Inter. Journal of Algorithms, Computing and Mathematics, 2(3) (2009) 107 - 116. 